\title{
ESTADO NUTRICIONAL, PERCENTUAL DE GORDURA E ASPECTOS DIETÉTICOS DE CRIANÇAS E ADOLESCENTES COM SÍNDROME DE DOWN
}

Monique Stephanie Piovan Ribeiro, Thayná Souza Neves, Bianca Depieri Balmant

Universidade do Oeste Paulista - UNOESTE, Faculdade de Ciências da Saúde, Curso de Nutrição, Presidente Prudente/SP. E-mail: biancadepieribalmant@hotmail.com

\section{RESUMO}

Este estudo avaliou o estado nutricional e aspectos dietéticos de portadores de Síndrome de Down (SD), entre 5 e 18 anos, vinculados a uma associação para cuidados com pessoas excepcionais. A amostra foi composta por 15 portadores de Síndrome de Down, sendo que para avaliação do estado nutricional realizou-se medidas antropométricas (peso e altura) e verificou-se o percentual de gordura corporal (\%G) por meio de bioimpedância. Para avaliação de hábitos alimentares foi aplicado um questionário de frequência alimentar. Observou-se uma alta prevalência de excesso de peso e \%G elevado. O peso corporal e \%G foi maior nos indivíduos com consumo frequente de alimentos ricos em gordura e carboidrato, bem como menor, naqueles com consumo frequente de frutas, verduras e legumes. Conclui-se que a obesidade das crianças e adolescentes sindrômicas não está somente relacionada à alteração cromossômica, mas também ao hábito alimentar que os mesmos podem possuir.

Palavras-chave: Avaliação nutricional, obesidade, composição corporal, consumo alimentar, síndrome de Down.

\section{NUTRITIONAL STATUS, FAT PERCENTAGE AND DIETS OF CHILDREN AND ADOLESCENTS WITH DOWN SYNDROME}

\begin{abstract}
This study evaluated the nutritional status and dietary aspects of Down Syndrome (SD), between 5 and 18 years, linked to an association for the care of exceptional people. The sample consisted of 15 Down Syndrome patients, and anthropometric measurements (weight and height) were used to assess the nutritional status and the percentage of body fat (\%Fat) was verified through bioimpedance. A food frequency questionnaire was used to evaluate dietary habits. A high prevalence of overweight and high \%Fat was observed. Body weight and \%G were higher in individuals with frequent consumption of high fat and carbohydrate foods, as well as lower in those with frequent consumption of fruits, vegetables and vegetables. It is concluded that the obesity of syndromic children and adolescents is not only related to the chromosomal alteration, but also to the alimentary habits they may have.
\end{abstract}

Keywords: Nutritional assessment, obesity, body composition, food consumption, Down syndrome.

\section{INTRODUÇÃO}

A Síndrome de Down (SD) foi descoberta pelo médico inglês John Langdon Haydon Down em 1866, que exercia a profissão em uma clínica para crianças com atraso neuropsicomotor. $\mathrm{Na}$ época, Down notou características físicas que eram semelhantes entre alguns filhos de mães com mais de 35 anos $^{1}$.

Atualmente, sabe-se que a Síndrome de
Down é uma mutação genética e metabólica que pode ocorrer de três formas cromossômicas. A primeira delas é a trissomia livre do 21, caracterizada por uma falha genética que ocorre durante a meiose, onde são expressas três cópias do cromossomo 21, expressando um cariótipo $47 X Y$ no sexo masculino e 47XX no sexo feminino. Este tipo é o mais frequente, correspondendo a $95 \%$ dos casos $^{2}$. Pode ocorrer também a 
translocação, mecanismo onde o cromossomo normal perde seu braço curto, possibilitando que o material cromossômico extra fique aderido a ele, o que resulta em uma soma total de 46 cromossomos. Este tipo representa cerca de 3\% dos casos $^{3}$. Por último, pode haver o mosaicismo, que também é uma falha de não-disjunção na tentativa de corrigir o processo de meiose, porém afeta apenas uma parte das células subsequentes, onde algumas terão 46 cromossomos e outras 47 cromossomos. Esta forma é encontrada em apenas $2 \%$ dos casos diagnosticados ${ }^{5}$.

Além das características genéticas, a Síndrome de Down possui particularidades físicas, metabólicas e mentais. Os portadores apresentam defasagem no desenvolvimento mental em níveis leve (QI 50 a 70), moderado (QI 35 a 50 ) e grave (QI abaixo de 20$)^{5}$. Além disto, são características físicas de portadores de Síndrome de Down: hipotonia geral, braquicefalia ou cabeça achatada, cabelos lisos, castanhos e escassos, pálpebras pequenas, pele pouco elástica, orelhas de implantação baixa e aparência dobrada, língua grossa, boca aberta, manchas de Brushfield, pescoço e membros superiores e inferiores curtos, mãos e pés achatados e largos ${ }^{6}$.

Em relação às características metabólicas, o sistema endócrino é afetado pelo hipotireoidismo, tendo características similares com a doença, como a pele seca, bradicardia, excesso de peso, atividade lentificada, prejuízo mental e baixa estatura ${ }^{7}$. 0 atraso de crescimento em crianças e adolescentes com Síndrome de Down se inicia no período pré-natal, no qual ocorre uma disfunção no eixo hormônio de crescimento, observada por uma alteração secretória do hormônio $\mathrm{GH}$ que reflete no excessivo ganho de peso e na baixa necessidade energética, resultando no desenvolvimento de obesidade $^{8}$.

A cardiopatia congênita também é observada em 40 a $60 \%$ de crianças com Síndrome de Down, principalmente nos dois primeiros anos de vida, onde sua progressão pode impactar o sistema cardiovascular, acarretando insuficiência cardíaca, pneumonia, arritmia cardíaca e hipertensão pulmonar ${ }^{9}$. Nestes casos, observa-se um risco de desnutrição antes da intervenção cirúrgica, porém após a correção, os pais passam a ter atitude protetora, o que leva a uma inatividade física e consequentemente a estados de obesidade ${ }^{10}$. Além disto, indivíduos com Síndrome de Down apresentam frequentes disfunções patológicas e imunológicas, dentre elas: doença celíaca, constipação e doença do refluxo gastresofágico ${ }^{11}$.

Considerando todas estas alterações, a

Síndrome de Down tem como perfil antropométrico o ganho excessivo de peso ${ }^{12}$. Entretanto, a obesidade das crianças e adolescentes sindrômicas pode não estar somente relacionado à alteração cromossômica, mas também ao hábito alimentar e estilo de vida sedentário que os mesmos podem possuir ${ }^{4,13}$.

Acredita-se que crianças e adolescentes com Síndrome de Down estão cercados de hábitos alimentares inadequados que muitas vezes passam despercebidos ou são estimulados pelos pais. Por isso, este estudo tem como objetivo avaliar a composição corporal e aspectos dietéticos de portadores de Síndrome de Down, entre 5 a 18 anos de idade.

\section{MÉTODOS}

Estudo transversal com análise descritiva, realizado em portadores de Síndrome de Down com idade entre 5 e 18 anos, de ambos os sexos, vinculados a associação para cuidados com pessoas excepcionais.

Todas as crianças e adolescentes com Síndrome de Down da Associação ( $n=20)$, que se encaixavam na faixa etária do estudo, foram convidadas a participar, porém só foram incluídas aquelas cujo o responsável assinou o termo de consentimento livre e esclarecido (TCLE), resultando em um total de 15 participantes. Além disto, eram critérios de exclusão crianças ou adolescentes portadores de prótese metálica, e/ou com responsáveis incapazes de responder ao questionário de frequência alimentar e/ou cuja a coleta de dados não tenha sido completa.

Os dados foram coletados entre novembro de 2017 a março de 2018, após aprovação do Comitê de Ética para Pesquisa sob o número CAAE 69585517.3.0000.5515.

\section{Estado Nutricional e Percentual de Gordura}

$O$ estado nutricional dos participantes foi verificado através das curvas de crescimento de peso por idade $(P / I)$, altura para idade $(A / I)^{14}$ e índice de massa corporal para idade $(\mathrm{IMC})^{15}$, específico para portadores de Síndrome de Down. Para isso, foram aferidos peso através de balança digital (Mondial ${ }^{\circledR}$, graduação de $100 \mathrm{~g}$, 
capacidade de $150 \mathrm{~kg}$ ) e estatura com estadiômetro móvel (Sanny $\left.{ }^{\circledR}\right)$, com precisão de $0,1 \mathrm{~cm}$, seguindo o procedimento preconizado pela Organização Mundial da Saúde $(\mathrm{OMS})^{16}$.

Para avaliação do percentual de gordura, foi utilizado o Biodynamics Body Composition Analyzer 310e (Biodynamics Corporation Seattle ${ }^{\circledR}$ ), que fornece os resultados através da determinação da impedância ao fluxo da corrente entre a fonte e os eletrodos detectores de voltagem e da conversão desta em densidade corporal, através da adição de dados referentes ao peso, estatura, sexo e idade. Os resultados apresentados são baseados em equações de regressão que correlacionam o método da bioimpedância elétrica com o método de pesagem hidrostática em tanque de flutuação ${ }^{17}$.

Para correta determinação, os participantes se posicionaram em decúbito dorsal, em posição confortável e relaxada, com os membros superiores ao longo do corpo e os inferiores em extensão, mantendo uma distância aproximada de 15 a $20 \mathrm{~cm}$ entre os mesmos. 0 membro superior direito foi posicionado a aproximadamente $15 \mathrm{~cm}$ do tronco, com punho em posição neutra, apoiado na maca e dedos em extensão, para que na região dorsal da mão, o eletrodo distal injetor de corrente pudesse ser posicionado na base da terceira falange proximal e o eletrodo proximal detector de voltagem no eixo da articulação rádio-cárpica, entre o processo estiloide da ulna e do rádio. Com o membro inferior direito relaxado, o eletrodo distal injetor de corrente foi posicionado na região dorsal do pé, na base da terceira falange proximal e o eletrodo proximal detector de voltagem na linha da articulação do tornozelo, face anterior, entre os maléolos interno e externo ${ }^{18}$.

A classificação do percentual de gordura $(\% \mathrm{G})$ foi realizada através da proposta de Lohman $^{19}$, específico para a idade do grupo estudado.

\section{Avaliação Dietética}

Para avaliação de hábitos alimentares, optou-se por utilizar um questionário de frequência alimentar (QFA) específico para a idade, validado na literatura científica, que se baseia no princípio de que a análise da média de ingestão ao longo de um período maior de tempo (consumo de semanas, meses ou anos), representa conceitualmente uma exposição mais importante do que o consumo de alguns dias específicos, fornecendo assim um consumo alimentar habitual $^{20}$.

Este QFA é composto por 6 questões, sendo distribuídas da seguinte forma:

- Questão 1 refere-se a mudanças de hábitos alimentares ou perda de peso voluntaria.

- Questão 2 divide-se em grupos de alimentos: sopas e massas, carnes e peixes, leite e derivados, leguminosas e ovos, arroz e tubérculos, verduras e legumes, molhos e temperos, frutas, bebidas, pães e biscoitos, doces e sobremesas.

- Questões 4 e 5 refere-se aos métodos de preparações.

- Questão 6 questiona os locais e horários que costuma fazer as refeições.

A aplicação deste questionário foi feita com o responsável na forma de entrevista, na qual os mesmos escolheram a frequência do consumo de cada alimento e o tamanho da porção do consumo (pequena, média, grande e extragrande) e as pesquisadoras assinalaram.

\section{Análise Estatística}

Primeiramente foi realizada uma análise descritiva através do cálculo de frequências e construção de gráficos. As variáveis quantitativas foram representadas através de medidas numéricas como: média, desvio-padrão e coeficiente de variação.

Para análise do QFA, foi adotada uma estratégia de atribuição de peso para as respostas qualitativas, ou seja, a unidade e a porção:

\begin{tabular}{cc}
\hline Porção & Peso \\
\hline E & 4 \\
G & 3 \\
M & 2 \\
P & 1 \\
\hline Unidade & Peso \\
\hline D & 4 \\
S & 3 \\
M & 2 \\
A & 1 \\
\hline
\end{tabular}

Portanto, na Unidade o peso maior foi atribuído à Diariamente, que é a maior frequência, e na Porção o peso maior foi atribuído à Extragrande, que é a maior porção. Depois de atribuir os pesos, foi realizada a 
seguinte multiplicação para cada criança: Quantas vezes come x Unidade x Porção. 0 resultado mínimo para essa multiplicação é 0 , indicando que a criança nunca come esse alimento, e quanto maior a multiplicação, maior a frequência de consumo do alimento.

Após, foi calculado o Coeficiente de Correlação Linear de Pearson para verificar a existência de associação entre pares de variáveis.

\section{RESULTADOS}

Foram coletados dados de 15 portadores de Síndrome de Down, 7 meninas e 8 meninos, com média de idade de $12,7 \pm 4,7$ anos. A Figura 1 apresenta a distribuição da classificação do IMC dos participantes deste estudo, de acordo com a referência de Myrelid $^{15}$, onde observa-se que $59,99 \%$ deles apresentaram excesso de peso $(26,66 \%$ com sobrepeso e $33,33 \%$ com obesidade).

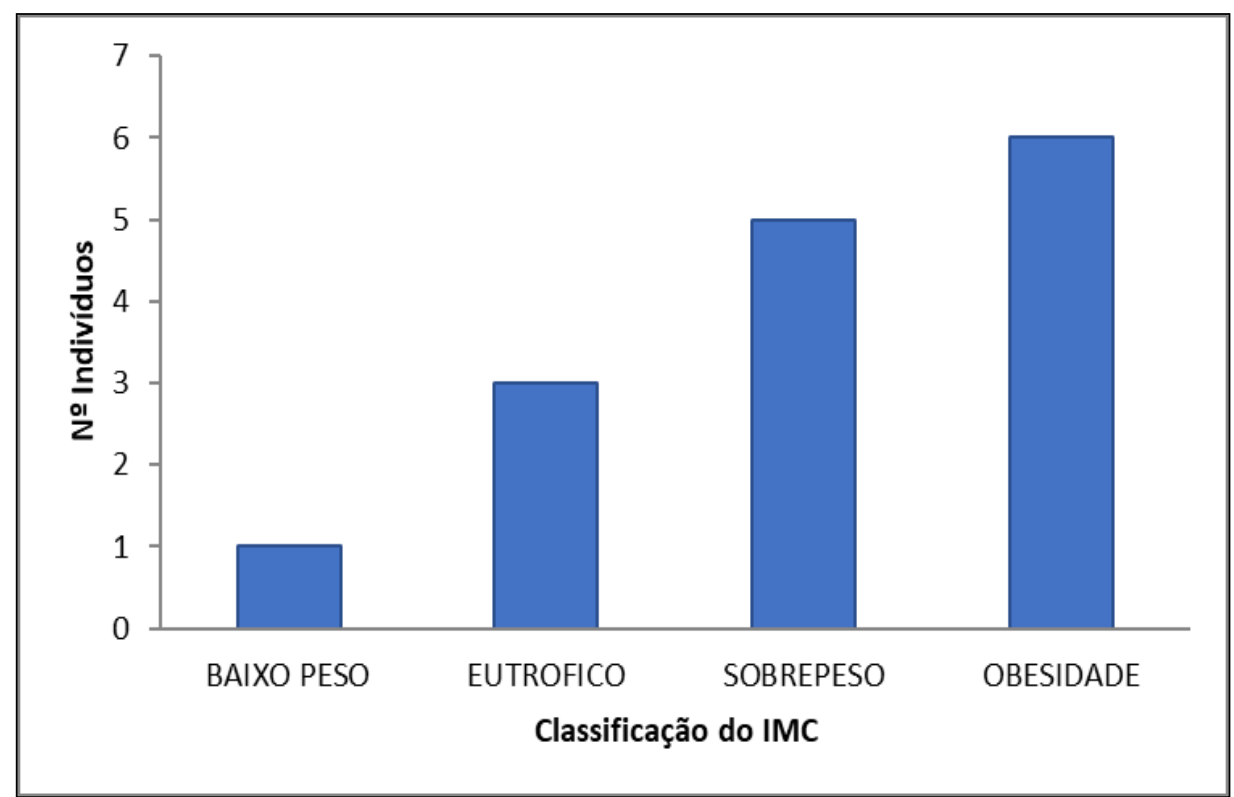

Figura 1. Estado nutricional pelo Índice de Massa Corporal (IMC) de portadores de Síndrome de Down, com idade entre 5 e 18 anos.

Em relação a $\mathrm{A} / \mathrm{I}$, verifica-se que $33,33 \%$ dos participantes apresentaram uma baixa estatura para idade, quando comparado com crianças e adolescentes também portadores de Síndrome de Down (Figura 2). Observa-se ainda, que $80 \%$ da amostra estudada, apresentou excesso de peso (percentil maior que 75) ao analisar 0 índice antropométrico $\mathrm{P} / \mathrm{I}$ pela referência de Cronk et $a^{14}{ }^{14}$ específica para esta população (Figura 3).

A Figura 4 mostra a distribuição do percentual de gordura $(\% G)$ das crianças e adolescentes estudados.
$\mathrm{Na}$ análise descritiva, observou-se que nenhuma das crianças ou adolescentes estudados praticava algum tipo de atividade física, assim como foi relatado pelos responsáveis, predomínio de atividades sentadas.

Em relação ao perfil alimentar, todos da amostra referem realizar a maior parte das refeições em casa. A maioria (73,3\%) não mudaram seus hábitos alimentares recentemente e não seguem nenhum tipo de dieta ou orientação nutricional. Da amostra, apenas 4 crianças mudaram seus hábitos alimentares recentemente (há menos de um mês) para perda de peso, sem supervisão do nutricionista. 


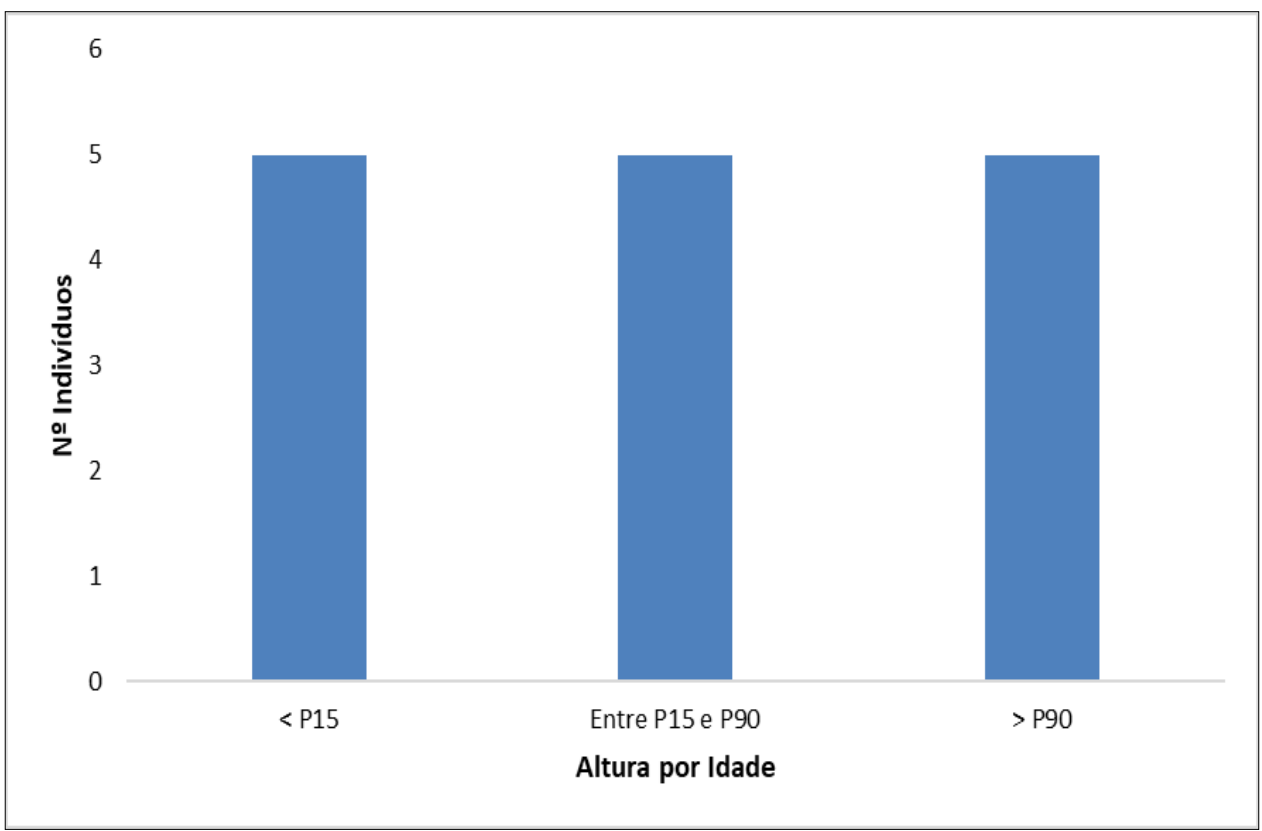

Figura 2. Altura por Idade de portadores de síndrome de Down, com idade entre 5 e 18 anos.

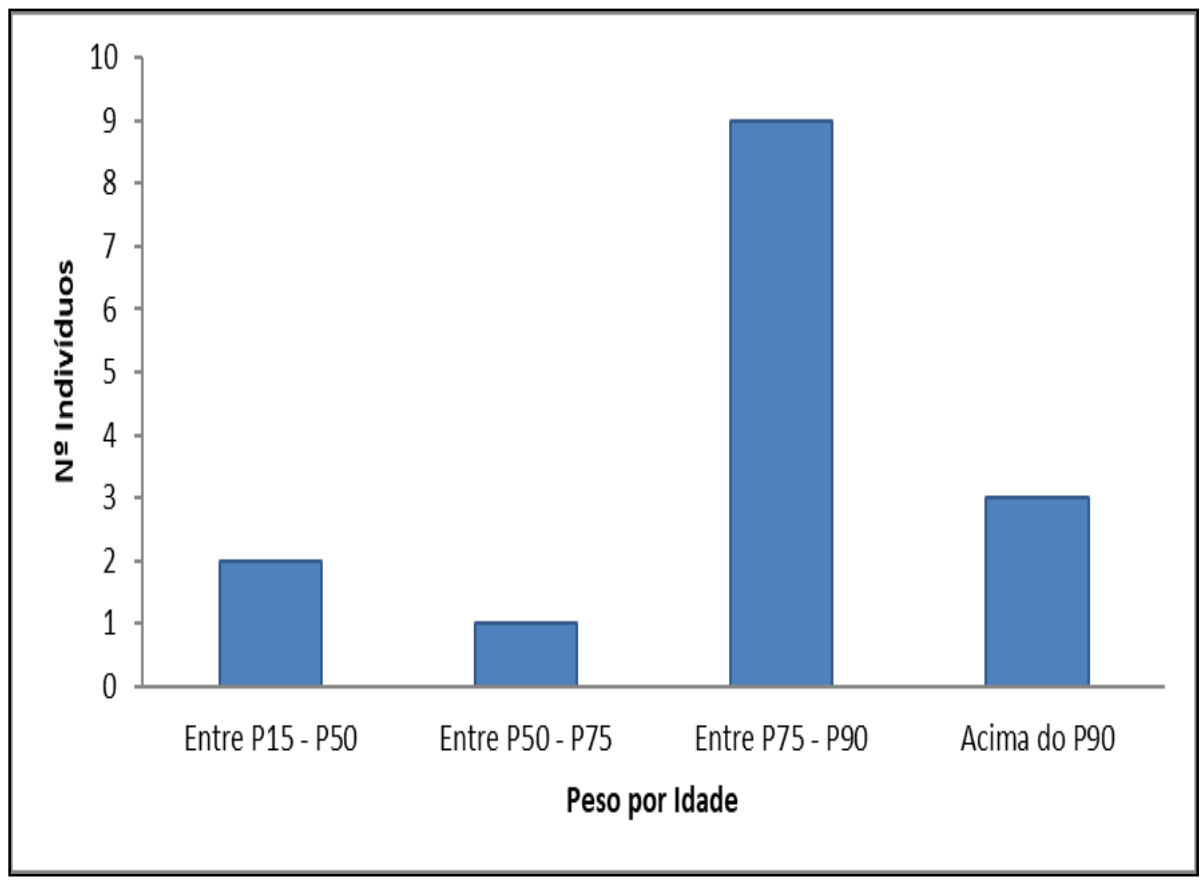

Figura 3. Peso por Idade de portadores de síndrome de Down, com idade entre 5 e 18 anos. 


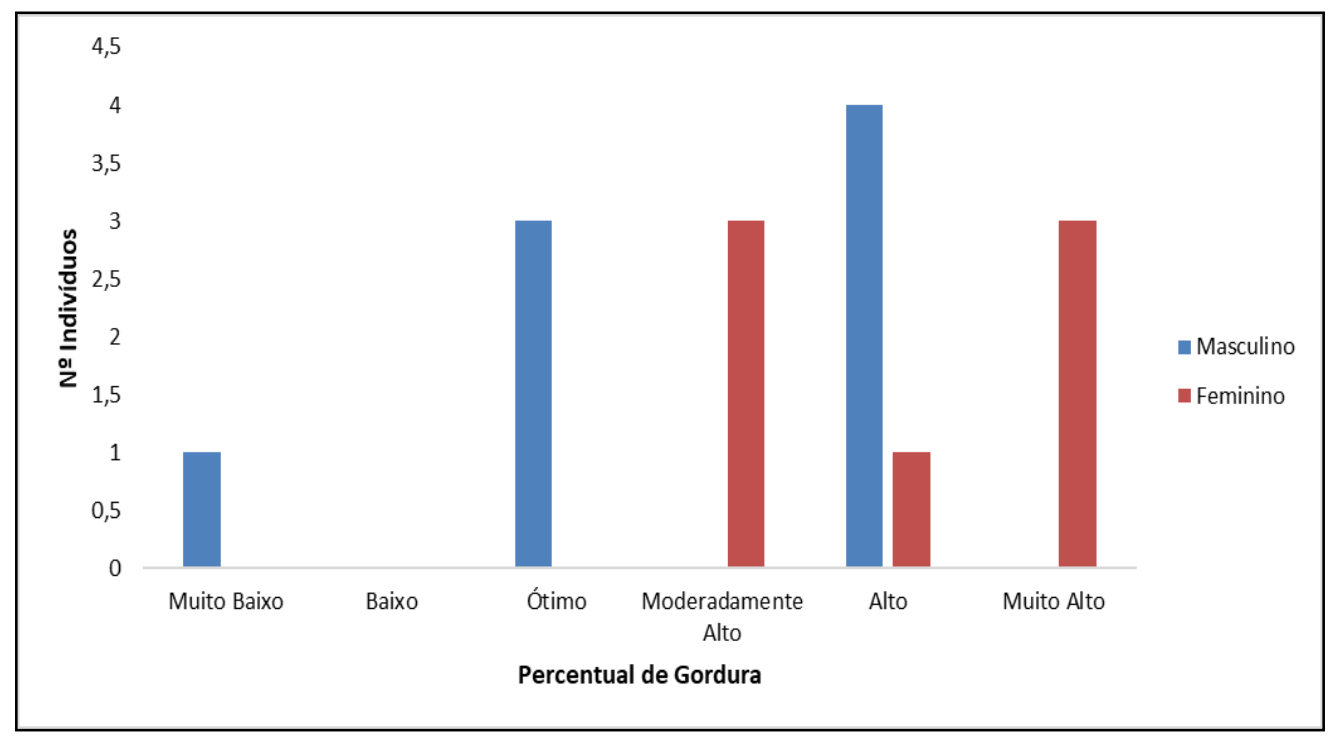

Figura 4. Distribuição do percentual de gordura $(\% \mathrm{G})$ de portadores de síndrome de Down, com idade entre 5 e 18 anos, em ambos os sexos.

A respeito dos métodos de preparação, $86,6 \%$ das crianças e adolescentes referiram não consumir a gordura visível da carne e nem a pele do frango ou peru.

Os resultados da multiplicação das variáveis do QFA, para os grupos de alimentos, estão dispostos na Tabela 1, correspondendo a uma média de consumo pela amostra.

Tabela 1. Média da atribuição de peso do consumo alimentar por grupo de alimentos de portadores de síndrome de Down, com idade entre 5 e 18 anos.

\begin{tabular}{lc}
\hline Grupo de Alimentos & Média \pm DP \\
\hline Arroz e Tubérculos & $18,4 \pm 9,0$ \\
Molhos e Temperos & $17,8 \pm 11,6$ \\
Pães e Biscoitos & $17,1 \pm 13,0$ \\
Leguminosas e Ovos & $16,2 \pm 11,8$ \\
Frutas & $12,8 \pm 11,1$ \\
Verduras e Legumes & $11,5 \pm 8,8$ \\
Bebidas & $11,3 \pm 9,0$ \\
Doces e Sobremesas & $10,6 \pm 9,3$ \\
Carnes e Peixes & $10,4 \pm 5,8$ \\
Leite e Derivados & $10,4 \pm 8,7$ \\
\hline
\end{tabular}

A Tabela 2 apresenta a correlação testada entre a variável peso e a ingestão de determinados alimentos.

\section{DISCUSSÃO}

A análise dos resultados desse estudo permitiu verificar a alta prevalência de excesso de
Tabela 2. Correlação entre peso e consumo alimentar de determinados alimentos de portadores de síndrome de Down, com idade entre 5 e 18 anos.

\begin{tabular}{lc}
\hline Alimento & Correlação \\
\hline Óleo/ Azeite & $0,71^{*}$ \\
Sal & 0,44 \\
Frutas & $0,78^{*}$ \\
Carnes & 0,5 \\
Leite Integral & 0,21 \\
Legumes/Verduras & $-0,73^{*}$ \\
Salgado Frito & 0,36 \\
Feijão & 0,43 \\
Manteiga & 0,38 \\
Pão/ Biscoitos & $0,69^{*}$ \\
Líquidos com açúcar & $0,71^{*}$ \\
Correlação moderada a forte &
\end{tabular}

Nos casos em que a correlação tem um sinal negativo, percebe-se que quanto mais o consumo desse tipo de alimento, menor é o peso. Nas correlações positivas, verifica-se que quanto maior o consumo desses alimentos, maior é o peso da criança ou adolescente. 
estudos anteriores. Dal Bosco et al. ${ }^{21}$, também encontraram uma prevalência de excesso de peso similar, sendo $30,4 \%$ para obesidade e $26,1 \%$ de sobrepeso na população com Síndrome de Down. Outros estudos identificaram percentuais ainda maiores de sobrepeso e obesidade ${ }^{22,23}$, reafirmando que crianças e adolescentes com Síndrome de Down têm uma maior tendência a desenvolver execesso de peso.

WhittGlover et al. ${ }^{24}$ aponta em seu estudo, que a atividade física intensa em crianças com Síndrome de Down pode prevenir a obesidade. Entretanto, observa-se na literatura que a inatividade física é comum nesta população ${ }^{21}$, por dinfunções patológicas e por ausencia de incentivo a prática ${ }^{10,11}$. Na amostra deste estudo, as crianças e adolescentes apresentaram-se sedentarias de acordo com relato dos responsáveis, favorecendo ainda mais a prevalência alarmante de excesso de peso.

Observou-se ainda neste estudo, percentuais de gordura alto em ambos os sexos, com prevalência principalmente no sexo feminino. Segundo Alaniz et al. ${ }^{25}$ esta diferença entre os sexos justifica-se pelo dimorfismo sexual na distribuição do tecido adiposo, na qual em mulheres é acentuado. Em um estudo que avaliou a \%G pelas pregas cutâneas, também foi encontrado percentuais altos de gordura, com diferença estatística entre os sexos ${ }^{26}$. Os achados deste estudo também estão de acordo com o realizado por González-Agüero et al. $^{27}$, que investigaram as diferenças de massa gorda e magra entre meninos e meninas com Síndrome de Down.

Vale ressaltar que pesquisas acerca da composição corporal de portadores de Síndrome de Down são escassos, sendo o público adulto, geralmente, o foco principal destes estudos. Além disso, embora a classificação do estado nutriconal seja feita através de curvas específicas para este grupo, uma vez que o processo de crescimento e tendencia à obesidade difere muito da população geral $\left.\right|^{28}$, as amostras utilizadas para o seu desenvolvimento foram relativamente pequenas, além de que no Brasil, por ser um país com grande diferença social, populações com baixa renda estão propensas a uma restrição no crescimento devido a subnutrição ${ }^{29}$, o que nos leva a questionar a eficácia de tais métodos.

Ao analisar os dados de A/I, foi constatado maior incidência de estatura adequada para idade, embora $33,33 \%$ da amostra apresente baixa estatura. Estes dados estão fortemente relacionados à síndrome, devido as caracteristicas metabólicas como o hipotireoidismo e a disfunção do hormônio $\mathrm{GH}$ que resulta na alteração secretória desse hormônio ${ }^{8}$. Resultados semelhantes foram entrados por Mustacchi etal. ${ }^{30}$, ao avaliar 350 crianças e adolescentes com Síndrome de Down através dos índices antropométricos.

Em relação ao perfil alimentar, verifica-se neste estudo o alto consumo de alimentos ricos em carboidratos como arroz, pão e acúcares simples, associado ao consumo de óleos/frituras, molhos e condimentos. Embora seja relatado pelos responsáveis o consumo frequente de frutas e verduras, percebe-se que há predileção a alguns alimentos, como banana, laranja, alface e tomate.

Estudos demonstram que crianças com Síndrome de Down preferem consumir alimentos com carboidratos simples e limitam o consumo de frutas frescas e vegetais, resultando dentre outras complicações, a baixa ingestão de fibra dietética $^{13,31}$. Em um estudo feito por Claretta e Chiorzi $^{13}$, foi observado que crianças com Síndrome de Down apresentam preferências por alimentos altamente calóricos, ricos em gordura e açúcares, corroborando para o perfil encontrado na amostra deste estudo.

O baixo consumo de doces e sobremesas relatado pelos responsáveis das crianças e adolescentes com Síndrome de Down pode estar relacionado as limitações deste estudo, uma vez que pode ocorrer omissão de consumo de alimentos durante a aplicação do questionário.

Através do QFA, verificou-se também um alto consumo de sal pelos participantes deste trabalho. Estudos também revelam um alto percentual de crianças e adolescentes com Síndrome de Down superando a ingestão dietética de sódio, denotando um comportamento preocupante neste grupo, uma vez que portadores de Síndrome de Down apresentam aumento da incidência de doenças cardiovasculares ${ }^{32-34}$.

Ao correlacionar o peso com os alimentos mais frequentes no habito alimentar de crianças e adolescentes deste estudo, constatou-se que quanto maior o consumo de alimentos como Óleos, Pães e Biscoitos e Líquidos com Açúcar, maior é o peso dos portadores de Síndrome de Down, provavelmente por serem alimentos com 
altos valores calóricos e estarem presentes com frequência na alimentação dessas crianças. Em contrapartida, notou-se que quanto maior o consumo de frutas, legumes e verduras pelas crianças e adolescentes, menor é o peso corporal dos avaliados.

Diante disto, realça-se a necessidade de orientação e acompanhamento nutricional para as crianças e adolescente, mas principalmente aos familiares, já que o ambiente familiar possui extrema importância para a educação nutricional e é a partir dele que se formam os hábitos alimentares, onde os vínculos são criados e onde o aprendizado de hábitos sociais e comportamentais é estabelecido. Essa intervenção se faz ainda mais necessária durante a infância e adolescência, pois crianças obesas tendem a se tornar adultos obesos e consequentemente podem vir a desenvolver doenças crônicas não transmissíveis na vida adulta.

Conclui-se que, em ambos os sexos, a prevalência de sobrepeso e obesidade, associada a percentuais elevados de gordura, em crianças e adolescentes com Síndrome de Down é preocupante e evidencia a importância da

\section{REFERÊNCIAS}

1. Silva NLPS, Dessen MA. Síndrome de Down: etiologia, caracterização e impacto na família. Inter Psic. 2002;6(2):167-76. DOI: https://doi.org/10.5380/psi.v6i2.3304

2. Gorla JI, Duarte E, Costa LT, Freire F. Crescimento de crianças e adolescentes com Síndrome de Down: uma breve revisão de literatura. Rev Bras Cineantrop. 2011;13(3):230-37.

3. Prado MB, Mestrinheri L, Frangella VS, Mustacchi Z. Acompanhamento nutricional de pacientes com síndrome de Down atendidos em um consultório pediátrico. Mund Saúd. 2009;33(3):335-46.

4. Santos JA; Franceschini SCC, Priore SE. Curvas de crescimento para crianças com síndrome de Down. Rev Bras Nutri Clin. 2006;21(2):144.

5. Benghi RAC, Krambeck WM, Lobe MCSB. Reconhecimento e confirmação diagnóstica. In: Fogaça HR, Lobe MCS. Síndrome de Down manejo e atenção clínica. Nov Letr. 2011:65-7.

6. Henn CG, Piccinini CA, Garcias GL. A família no contexto da Síndrome de Down: revisando a intervenção por parte de órgãos e profissionais da área da saúde neste grupo de índivíduos.

Conclui-se ainda, que a má qualidade alimentar identificada pelo questionário de frequência alimentar se relaciona diretamente com a incidência de sobrepeso e obesidade e que a presença de nutricionista em escolas, associações e ambulatorios de atendimento para esse público, pode ser um meio para reduzir tais índices, pois contribuir para o atendimento das necessidades nutricionais, bem como para a formação de hábitos alimentares saudáveis.

Sugere-se que novos estudos acerca desta temática seja feito, de forma a englobar amostras populacionais maiores e medidas de controle de ingestão habitual mais rigososas.

\section{AGRADECIMENTOS}

À Universidade do Oeste Paulista UNOESTE pelo financiamento desta pesquisa.

\section{CONFLITO DE INTERESSE}

Os autores declaram não haver qualquer potencial de conflito de interesse que possa interferir na imparcialidade deste trabalho científico.

literatura. Psic Estud. 2008; 13(3):485-533. DOI: https://doi.org/10.1590/S1413-73722008000300009

7. Moura AB, Mendes A, Peri A. Passoni CRMS. Aspectos nutricionais em portadores da Síndrome de Down. Cadern Escol Saúd. 2009;2:1-11.

8. Reuter C, Roskowski LC, Lobe MCS. Crescimento. In: Fogaça HR, Lobe MCS. Síndrome de Down manejo e atenção clínica. Nov Letr. 2011:73-8.

9. Mourato FA, Villachan LRR, Mattos SS. Prevalência e perfil de cardiopatias congênitas e hipertensão pulmonar na síndrome de Down em serviço de cardiologia. Rev Paul Pediatr. 2014;32(2):159-63. DOI: https://doi.org/10.1590/0103-0582201432218913

10. Sica CA, Cesa CC, Pellanda LC. Curvas de crescimento na síndrome de Down com cardiopatia congênita. Rev Ass Méd Bras. 2016;62(5):414-20. DOI: https://doi.org/10.1590/1806-9282.62.05.414

11. Gios DR, Rosa KZ, Vargas DM. Avaliação Gastrointestinal. In: Fogaça HR, Lobe MCS. Síndrome de Down manejo e atenção clínica. Nov Letr. 2011:157-67. 
12. Wanderley EM, Ferreira VA. Obesidade: uma perspectiva plural. Ciênc Saúd Colet. 2010;15(1):18594. DOI: https://doi.org/10.1590/S1413$\underline{81232010000100024}$

13. Claretta A, Ghiorzi AR. O ato de comer e as pessoas com Síndrome de Down. Rev Bras Enferm. 2009;62(3):480-84. DOI: https://doi.org/10.1590/S0034-71672009000300024

14. Cronk C, Crocker AC, Pueschel SM, Shea AM, Zackai E, Pickens $G$, et al. Growth charts for children with Down syndrome: 1 month to 18 years of age. Pediatrics. 1988;81(1):102-10.

15. Myrelid A. Growth charts for Down's syndrome from birth to 18 years of age. Arch Dis Child. 2002;87:97-103. DOI:

https://doi.org/10.1136/adc.87.2.97

16. Brasil. Ministério da Saúde (MS). Vigilância alimentar e nutricional - Sisvan: orientações básicas para a coleta, processamento, analise de dados e informação em serviços de saúde - Brasília: Ministério da Saúde, 2011.

17. Freedman DS, Perry G. Body composition and health status among children and adolescents. Prevent Med. 2000;31:34-53.

DOI:

https://doi.org/10.1006/pmed.1998.0480

18. Sung RYT, Sung $R$, Lau $P, Y u$ C, Lam $P$. Measurement of body fat using leg to leg bioimpedance. Arch Dis Child. 2001;85:263-7. DOI: https://doi.org/10.1136/adc.85.3.263

19. Lohman TG. The use of skinfold to estimate body fatness on children and youth. JOPERD. 1987;58(9):98102. DOI:

https://doi.org/10.1080/07303084.1987.10604383

20. Voci SM, Enes CC, Slater B. Validação do questionário de frequência alimentar para adolescentes (QFAA) por grupos de alimentos em uma população de escolares. Rev Bras Epidemiol. 2008;11(4):561-72. DOI: https://doi.org/10.1590/S1415-790X2008000400005

21. Dal Bosco SM, Scherer F, Altevogt CG. Estado nutricional de portadores de síndrome de Down no Vale do Taquari-RS. ConScientiae Saúde. 2011;10(2):278-84. DOI: https://doi.org/10.5585/conssaude.v10i2.2291

22. Shabayek MM. Assessment of the nutritional status of children with special needs in Alexandria: Part I. Nutrient intake and food consumption. J Egyp Public Health Assoc. 2004;79(3-4):225-41.
23. Dalpicolo F, Viebig RF, Nacif MAL. Avaliação do estado nutricional de crianças com Síndrome de Down. Nutr Brasil. 2004;6(1):336-40.

24. Whitt-Glover MC, O'Neill KL, Stettler N. Physical activity patterns in children with and without Down syndrome. Pediatr Rehabil. 2006;9(2):158-64. DOI: https://doi.org/10.1080/13638490500353202

25. Alaniz MHF, Takada J, Vale MICA e Lima FB. O tecido adiposo como centro regulador do metabolismo. Arq Bras Endocrinol Metab. 2006;50(2):221.

26. Freire F, Costa LT, Gorla JI. Indicadores de obesidade em jovens com Síndrome de Down. Motricidade. 2014;10(2):2-10. DOI: https://doi.org/10.6063/motricidade.10(2).1196

27. González-Agüero A, Ara I, Moreno LA, VicenteRodríguez G, Casajús JA. Fat and lean masses in youths with Down syndrome: Gender differences. Res Develop Disab. 2011;32:1685-93. DOI: https://doi.org/10.1016/j.ridd.2011.02.023

28. Bravo-Valenzuela NJM, Passarelli MLB, Coates MV. Curvas de crescimento pôndero-estatural em crianças com síndrome de Down: uma revisão sistemática. Rev Paul Pediatr. 2011;29(2):261-9. DOI: https://doi.org/10.1590/S0103-05822011000200019

29. Bertapelli F. Curvas de referência de crescimento para crianças e adolescentes com síndrome de down com idade entre 0 e 20 anos. [Tese de doutorado]. Faculdade de Ciências Médicas da UNICAMP, Campinas. 2016. 119p.

30. Mustacchi Z. Curvas padrão pôndero-estatural de portadores de Síndrome de Down procedentes da região urbana da cidade de São Paulo. [Tese de doutorado]. Faculdade de Ciências Farmacêuticas da USP, São Paulo. 2002. 210p.

31. Mazurek D, Wyka J. Down syndrome - genetic and nutritional aspects of accompanying disorders. Roczniki Pan'stwowego Zakładu Higieny. 2015;66:189194.

32. Alexander $M$, Petri $H$, Ding $Y$. Morbidity and medication in a large population of individuals with Down syndrome compared to the general population. Develop Med Child Neurology. 2016;58:246-54. DOI: https://doi.org/10.1111/dmcn.12868

33. Suckling RJ and Swift PA. The health impacts of dietary sodium and a low-salt diet. Clin Med. 2015;15:585-588. DOI: 
34. Barata LV, Branco A. Os distúrbios fonoarticulatóeios na Síndrome de Down e a intervenção precoce. Rev CEFAC. 2010;12(1):134. DOI: https://doi.org/10.1590/S1516-18462010000100018
Recebido para publicação em 08/08/2018 Revisado em 04/09/2018

Aceito em 09/09/2018 